\title{
Snake-3D na Modelagem Tridimensional e Medição do Comprimento de Arcos Elétricos
}

\author{
Gilmário B. Santos ${ }^{1}$ \\ Clésio L. Tozzi ${ }^{2}$ Maria C. Tavares ${ }^{3}$
}

Resumo: É proposta uma abordagem inédita baseada na aplicação de um contorno ativo tridimensional (snake-3D) no rastreio e medição do comprimento de arcos elétricos artificialmente gerados e capturados através de um par de câmeras calibradas. A snake-3D é representada por uma $B$-spline que evolui sob as restrições de forças externas ao contorno ativo e internas a este. As forças externas são obtidas a partir dos pares de imagens da evolução do arco elétrico. Neste trabalho os arcos elétricos apresentam alongamento expressivo e são produzidos em um laboratório de alta tensão a céu aberto utilizando torres reais. Esta é uma situação desfavorável à adaptação de outros trabalhos que aplicam técnicas de análise de imagens de arcos, os quais não têm por objetivo a medição em questão e aplicam estratégias bastante atreladas aos dispositivos e ambientes utilizados para a geração dos arcos. Por outro lado, a estratégia aqui apresentada se diferencia por uma maior adaptabilidade especialmente no posicionamento de câmeras, além de praticamente ser independente de métodos para determinação de homólogos. Para fins de avaliação foram realizados experimentos sobre imagens sintéticas obtidas de curvas analíticas, bem como experimentos com as imagens dos arcos elétricos reais produzidos artificialmente. Os resultados obtidos foram considerados aceitáveis, demonstrando o potencial da abordagem apresentada.

\footnotetext{
${ }^{1}$ Centro de Ciências Tecnológicas (CCT), Departamento de Ciências da Computação (DCC), Universidade do Estado de Santa Catarina (UDESC), Joinville-SC

\{gilmario@joinville.udesc.br\}

${ }^{2}$ Faculdade de Engenharia Elétrica e de Computação (FEEC), Universidade Estadual de Campinas (UNICAMP), Campinas-SP

\{clesio@dca.fee.unicamp.br\}

${ }^{3}$ Faculdade de Engenharia Elétrica e de Computação (FEEC), Universidade Estadual de Campinas (UNICAMP), Campinas-SP

\{cristina@dsce.fee.unicamp.br\}
} 


\begin{abstract}
It is proposed a novel approach based on a three-dimensional active contour (3D-snake), for the tracking and measurement of the length of artificially generated electrical arcs captured by a calibrated pair of cameras. The 3D-snake is represented by a $B$-spline which evolves in 3D space constrained by internal and external forces. The external forces come from pairs of images of the arc in evolution. In this work the electrical arcs present significant elongation and are produced in a high voltage laboratory in an open sky experiment with real scale power towers. This is an unfavorable situation for the adaptation of other works that apply techniques of image analysis of electric arcs, which are not aimed at the measurement in question and apply strategies rather tied to devices and environments used for arcs generation. On its turn, the strategy presented in this paper is distinguished by greater adaptability specially about the cameras positioning, additionally, it is practically independent on methods for the homologous points determination. For evaluation the proposed approach it was done experiments using synthetic images obtained from analytical curves as also using real images of the electrical arcs artificially generated. The results were considered acceptable verifying the potential of the proposed approach.
\end{abstract}

\title{
1 Introdução
}

As recomendações da Agência Nacional de Energia Elétrica (ANEEL) acerca do projeto de novas linhas de transmissão têm suscitado pesquisas visando a modelagem de arcos elétricos, sendo o comprimento do arco um importante parâmetro nessa modelagem. Em Camara $[1,2,3]$ é discutida a importância do alongamento no modelo do arco elétrico bem como o modelo propriamente dito. Ademais, em Portela [4], Anke et al. [5], Portela et al. [6, 7, 8] e Tavares et al. [9] encontram-se outros trabalhos relacionados à modelagem em questão.

Existem meios para estimar o alongamento do arco a partir de medições elétricas. Os sinais resultantes destas medições são analisados e feita a estimativa da curva $l(t)$ que descreve a variação do comprimento do arco no decorrer do tempo. Como procedimento complementar, os técnicos também inspecionam visualmente as imagens do ensaio buscando confirmar a coerência dos resultados da análise dos sinais elétricos. O problema desta abordagem é que os resultados obtidos não têm uma relação direta com comprimento do arco, estes não decorrem da medição do seu eixo-médio longitudinal, portanto, é produzido apenas o perfil aproximado da desejada curva $l(t)$.

Por outro lado, os pesquisadores observaram que as imagens dos ensaios poderiam ser utilizadas não apenas para apoiar o alongamento estimado através da análise eletrotécnica. Utilizando um par de câmeras notou-se que seria possível recuperar a configuração tridimensional do arco através de técnicas de análise de imagens por computador. Consequentemente, a visão computacional e o processamento de imagens passaram a ser aplicados visando a determinação do alongamento do arco em unidades de comprimento. 
Um outro aspecto de interesse é que esta nova abordagem dispensa a inserção de fatores ambientais (a velocidade e direção do vento, por exemplo) no modelo, visto que a influência destes fatores já são captadas nas imagens. Portanto, todos os dados necessários à reconstrução tridimensional do arco residem nas imagens cuja qualidade depende basicamente do sistema de aquisição utilizado para capturá-las.

Apesar de não se voltarem ao problema da medição de descargas elétricas, autores como MacAlpine e Qiu [10], Qiu et al. [11], [12], Amarasinghe et al. [13] e Sellathurai [14], apresentam alguns resultados importantes no campo da análise de arcos elétricos com base em imagens. Porém, devido à característica fortemente $a d-h o c$ das técnicas descritas por estes autores revelou-se frustrante a tendência inicial de adaptar estas estratégias para a solução do problema aqui tratado. Estes trabalhos são voltados basicamente ao estudo de trajetórias de descargas elétricas curtas (comprimento não superior a 1,0 m) quase sem formação de ondulação, produzidas em ambiente confinado e controlado. Em termos da reconstrução tridimensional, em geral estes trabalhos aplicam a projeção ortográfica ao invés da projeção em perspectiva, o que consiste em uma grande simplificação.

Há também uma forte dependência de métodos explícitos para a determinação de pares de pontos homólogos e, principalmente, estes autores desenvolvem suas estratégias de forma totalmente dependente de aparatos específicos desenvolvidos para os ensaios realizados, com posicionamentos bastante restritos para as câmeras utilizadas. Tratam-se de experimentos típicos de bancada. Por exemplo, em MacAlpine e Qiu [10], Qiu et al. [11], [12], um prisma é utilizado no sistema de aquisição, em Amarasinghe et al. [13] três câmeras precisam ser rigorosamente posicionadas sobre os vértices de um triângulo e Sellathurai [14] considera a existência de características que não se apresentam nos arcos tratados neste trabalho. Sendo assim, tais estratégias não são adaptáveis a diferentes casos, particularmente aos tratados no presente artigo.

A abordagem proposta neste trabalho foi motivada pela disponibilidade de registros visuais e a falta de estratégias eficientes baseados em imagens para o tratamento do problema de medição do comprimento de arcos elétricos longos (comprimento inicial de 4,0 m) gerados em ambiente a céu aberto. Neste trabalho se propõe uma aplicação inovadora fundamentada no modelo snake-3D (contorno ativo tridimensional) para modelagem do eixo-médio longitudinal do arco elétrico guiado por forças decorrentes de pares de imagens processadas (Santos et al. [15], [16], e [17]). É importante ressaltar que, no melhor dos esforços, não foi encontrado na literatura nenhum trabalho que meça/rastreie arcos elétricos. Trata-se de uma abordagem inédita não afetada pelo problema da determinação de pontos homólogos e mais adaptável a diferentes situações, o que é conveniente para os casos abordados neste trabalho. Estes se diferenciam daqueles tratados na literatura pois são mais alongados, mais sinuosos e resultantes de ensaios realizados em campo aberto (ambiente não controlado) em instalações com torres de alta tensão reais. 
A reconstrução 3D propiciada pela abordagem proposta não ocorre por um processo convencional, ou seja, a execução de um procedimento específico que detecta pares de pontos homólogos os quais são retroprojetados ao espaço. O que se propõe é aplicar a snake-3D para rastreio e medição do comprimento do arco elétrico sem a necessidade de tratar o problema da determinação de pontos homólogos.

O artigo está organizado da seguinte forma, na Seção 2 é feita a descrição do modelo matemático da snake-3D. Na Seção 3 discute-se as etapas de processamento de imagens necessárias e aborda-se a aplicação do operador gradiente na estratégia de determinação das forças externas necessárias à aplicação do modelo proposto, enquanto que a Seção 4 é dedicada à inicialização do modelo snake-3D. Na Seção 5 é feita a descrição do ajuste e evolução espacial do contorno ativo, a estratégia de medição utilizada e o rastreio da evolução do arco. Aspectos de calibração de câmeras para obtenção das matrizes de projeção no ambiente do CEPEL (Centro de Pesquisa em Energia Elétrica) são tratados na Seção 6. Para executar a validação da abordagem foram realizados testes utilizando curvas analíticas, estes testes, bem como seus resultados, são discutidos na Seção 7. A seguir, na Seção 8, destaca-se um dos experimentos realizados no CEPEL com a aplicação da abordagem sobre imagens reais. Por fim, a Seção 9 é dedicada às conclusões.

\section{Snake-3D}

Similarmente ao modelo físico-inspirado bidimensional (Equação 1) proposto por Kass et al. [18], o contorno ativo tridimensional aqui aplicado também se baseia em um processo de minimização da energia total do sistema sob a ação de forças internas e externas ao contorno ativo. No caso da snake-3D é necessário um par de imagens tomadas em um certo instante para servir como fonte primária de forças externas (é possível adaptar a abordagem para um número maior de imagens). Estas forças de imagem têm que ser transformadas para o espaço de pontos de controle da $B$-spline que representa espacialmente a snake-3D.

Considere um certo par de imagens $\left(I_{1}^{t}, I_{2}^{t}\right)$ que capturou o arco em um dado tempo $t$ de sua evolução. O processo de minimização de energias ocorre de maneira que a $B$-spline é movida no espaço 3D sob o controle das forças envolvidas fazendo com que gradativamente a snake-3D seja ajustada à configuração apresentada pelo eixo-médio longitudinal do arco naquele tempo $t$, passado. Neste momento o sistema atinge um mínimo de energia e as projeções da snake-3D estarão ajustadas ao par de imagens $\left(I_{1}^{t}, I_{2}^{t}\right)$ em questão.

Note que o processo de minimização de energia produz o movimento da snake-3D. Em termos gerais este processo está embutido na Equação 2, onde $\mathbf{V}_{t}$ corresponde ao novo conjunto de pontos de controle determinado em função das forças internas $\mathbf{F}_{\text {int }}$, das forças externas no espaço dos pontos de controle $\mathbf{F}_{e x t}^{p c}$ e do próprio conjunto atual de pontos de controle $\mathbf{V}_{t-1}$. A Equação 2 será melhor detalhada na Seção 3.3. 


$$
\begin{gathered}
E_{\text {total }}=\int_{0}^{1} E_{\text {int }}(v(s))+E_{\text {ext }}(v(s)) d s . \\
\mathbf{V}_{t}=\mathcal{F}\left(\mathbf{F}_{\text {int }}, \mathbf{F}_{\text {ext }}^{p c}, \mathbf{V}_{t-1}\right) .
\end{gathered}
$$

As forças internas controlam a continuidade do contorno ativo e a sua rigidez (ou flexibilidade) regulando a tensão entre os pontos de controle da $B$-spline. No que se refere à formulação matemática estas são obtidas pela aplicação da matriz de rigidez $\mathbf{H}$ segundo a Equação 3, conforme Blake e Isard [19] e Cañero [20].

$$
\mathbf{H}=\frac{1}{\mathcal{L}} \sum_{\sigma=0}^{\mathcal{L}-1} \mathbf{G}_{\sigma}^{T} \mathbf{N}_{\sigma}^{S^{T}}\left(\alpha \mathbf{P}^{\prime}+\beta \mathbf{P}^{\prime \prime}\right) \mathbf{N}_{\sigma}^{S} \mathbf{G}_{\sigma}
$$

Onde:

- $\mathcal{L}$ corresponde ao número de vãos (spans) $(\sigma)$ no vetor de pontos de nós da $B$-spline que representa espacialmente a snake-3D;

- $\mathbf{N}_{\sigma}^{S}$ representa uma matriz associada a um vão, a qual é obtida pelo "algoritmo para cálculo das matrizes de vão para uma $B$-spline" descrito em Blake e Isard [24];

- $\mathbf{G}_{\sigma}$ é a matriz que seleciona um subconjunto de pontos de controle associados a determinado vão. O termo geral de uma matriz é descrito na Equação 4, segundo Cañero [20]. Observe que que $b_{\sigma}$ é calculado conforme Equação 5, onde $m$ é a multiplicidade no $i$-ésimo nó do vetor de nós e $d$ corresponde à ordem da $B$-spline.

$$
\begin{gathered}
\left(\mathbf{G}_{\sigma}\right)_{i j}= \begin{cases}1 & \text { se }, j-b_{\sigma}=i \\
0 & \text { caso contrário. }\end{cases} \\
\text { Onde: } b_{\sigma}=\left(\sum_{i=0}^{\sigma} m_{i}\right)-d
\end{gathered}
$$

As matrizes $\mathbf{P}^{\prime}$ e $\mathbf{P}^{\prime \prime}$ são utilizadas para cálculo das continuidades de primeira e segunda ordem da B-spline e são derivadas da matriz $\mathbf{P}$, a qual é decorrente do cálculo da norma de curvas (Blake e Isard [19]). De acordo com Cañero [20] as matrizes $\mathbf{P}^{\prime}$ e $\mathbf{P}^{\prime \prime}$ são obtidas através das Equações 6 e 7 respectivamente:

$$
\mathbf{P}^{\prime}= \begin{cases}0 & \text { se } i=1 \text { ou } j=1 ; \\ \frac{(i-1)(j-1)}{i+j-3} & \text { caso contrário. }\end{cases}
$$




$$
\mathbf{P}^{\prime \prime}= \begin{cases}0 & \text { se } i<3 \text { ou } j<3 \\ \frac{\left(2-3 i+i^{2}\right)\left(2-3 j+j^{2}\right)}{i+j-5} & \text { caso contrário. }\end{cases}
$$

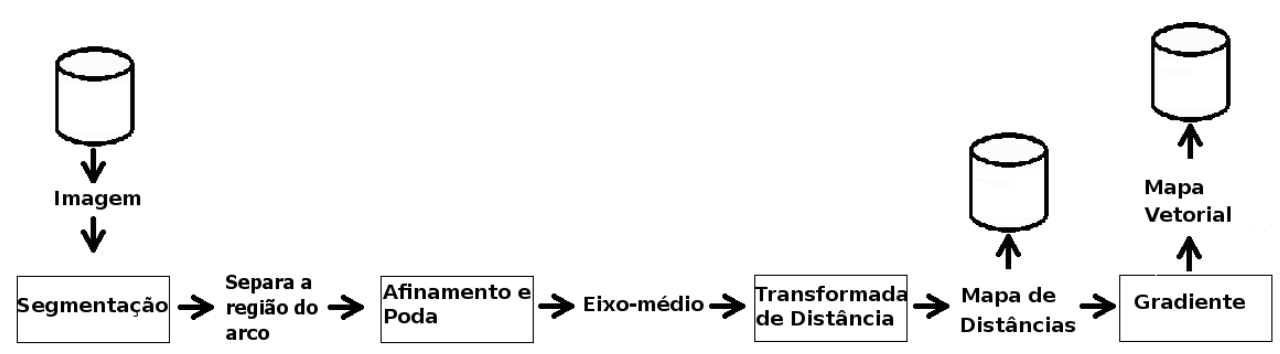

Figura 1. Sequência de processamento e transformações aplicadas em cascata com o objetivo de obter um mapa vetorial, todas as imagens são processadas desta forma.

\section{Determinação das Forças Externas}

As forças externas são essenciais para a correta aplicação do modelo proposto, para a determinação destas forças são realizadas as operações de processamento e transformações ilustradas na Figura 1, as quais serão descritas a seguir. Os mesmos passos são executados em cascata para cada imagem I disponível.

\subsection{Processamento de Imagens}

No modelo proposto, cada imagem $I$ é uma fonte primária de forças externas e precisa ser processada para evidenciação destas forças. O primeiro passo, para tanto, consiste em separar os quadros do par de vídeos do arco elétrico determinando uma sequência de pares de imagens em tons de cinza. Estas são processadas para a determinação do respectivo eixomédio longitudinal e para a evidenciação do campo de energia associado, a partir deste campo se extraem as forças decorrentes das imagens.

Para obter o eixo-médio é necessário segmentar a região do arco elétrico na imagem, o que é facilitado pelas características fotométricas do mesmo. Como se trata de um plasma de alta temperatura, o arco corresponde ao objeto mais brilhante na imagem (Figura 2-A) este efeito pode ser potencializado através do ajuste da "entrada de luz" pelos diafragmas das câmeras — o que viabiliza uma segmentação por limiarização seguida de filtragem por área (Gonzalez e Woods [21]), para eliminação de possíveis objetos selecionados erroneamente 
(A)

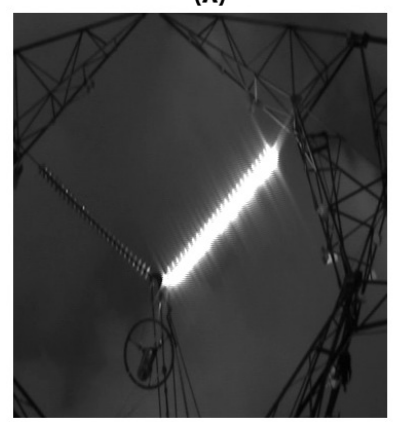

(B)

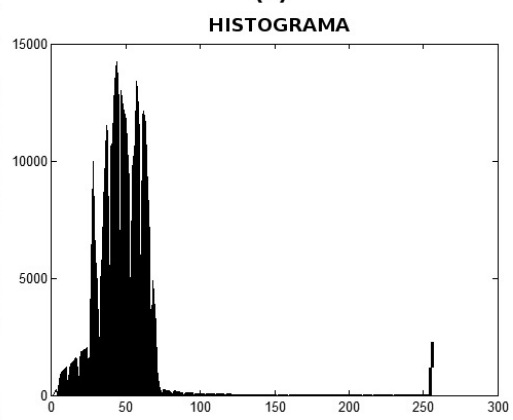

Figura 2. A) Imagem de um arco elétrico em seu instante inicial e B) seu histograma de brilho apresentando um vale entre duas elevações, os pixels do arco encontram-se na elevação correspondente aproximadamente ao valor de cinza $\approx 260$.

(Figura 3). A imagem resultante da segmentação/filtragem $\left(I_{\text {seg }}=\right.$ Segmenta $\left.(I)\right)$ é processada por afinamento e poda (Soille [22]) produzindo uma imagem resultante binária a qual contém o eixo-médio longitudinal do arco $\left(I_{\text {eixo }}=\right.$ AfinaPoda $\left.\left(I_{\text {seg }}\right)\right)$.

A determinação do limiar de segmentação é feita a partir do histograma da imagem $I$, o qual é tipicamente bimodal (Figura 2-B) tornando relativamente simples encontrar o limiar através da determinação, no histograma, do valor de cinza que separa a elevação referente aos pixels brancos. No caso ilustrado na Figura 2-B, este limiar corresponde a um valor próximo de 250 .

Nesta etapa é necessário evidenciar o campo de energia potencial associado à imagem binária do eixo-médio. A aplicação do gradiente diretamente sobre o eixo-médio produziria forças apenas nas bordas desse eixo. Neste caso o campo de forças de imagem não teria abrangência suficiente no entorno do eixo-médio, o que não seria suficiente para uma evolução ótima da snake-3D. Por outro lado, ao aplicar previamente a transformada de distâncias ${ }^{4}$ sobre a imagem do eixo-médio origina-se um amplo campo de energia externa, aqui chamado de mapa de distâncias $\left(I_{m d}=\operatorname{TranfDist}\left(I_{e i x o}\right)\right)$ o qual viabiliza uma resposta ao operador gradiente em uma região equivalente a toda a extensão da imagem. $\mathrm{O}$ resultado da aplicação do gradiente sobre o mapa de distâncias é designado como um mapa vetorial $\left(I_{m v}=\operatorname{Grad}\left(I_{m d}\right)\right)$ e corresponde ao campo de forças de imagem desejado.

A Figura 4-A exibe uma parte de um mapa de distâncias enquanto que a Figura 4-B

\footnotetext{
${ }^{4} \mathrm{~A}$ transformada de distâncias calcula a distância mínima entre cada pixel da imagem binária e o eixo-médio nela retratado
} 


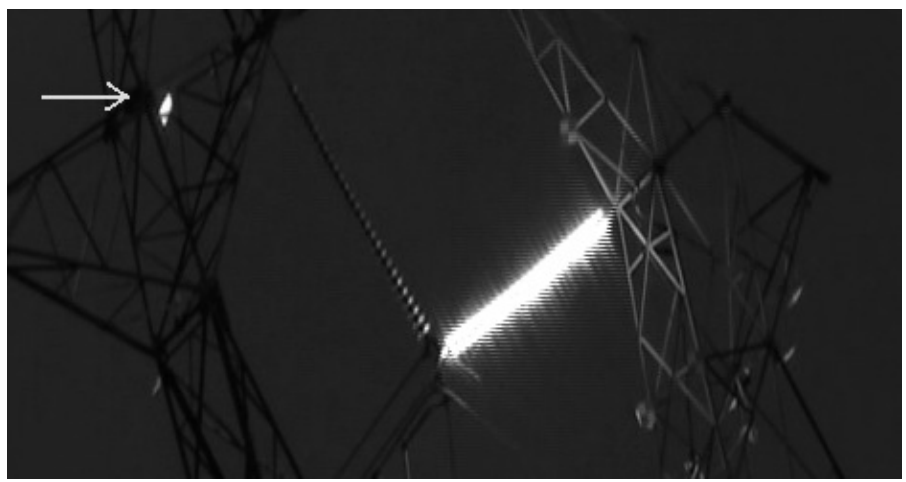

Figura 3. Um exemplo de objeto espúrio: o brilho refletido em uma placa de calibração será removido por segmentação por área.

exibe um detalhe do mapa vetorial obtido. O procedimento para obtenção do mapa vetorial de uma imagem foi exibida na Figura 1 e é descrito na Equação 8.

$$
\left.I_{m v}=\operatorname{Grad}(\operatorname{Trans} f \operatorname{Dist}(\text { AfinaPoda }(\operatorname{Segmenta}(I))))\right)
$$

\subsection{Obtenção da Força Externa 3D no Espaço dos Pontos de Controle}

Considerando a ilustração na Figura 5, os vetores de força de imagem serão designados como $F_{\text {ext } 2 D}$ e a composição tridimensional $F_{\text {ext } 3 D}$ decorre de um par de vetores $F_{\text {ext } 2 D}$ em mapas vetoriais distintos.

Uma das características importantes da $B$-spline consiste no controle local exercido através dos seus pontos de controle, o que confere maior flexibilidade à snake-3D. Para as forças de imagem exercerem influência sobre os pontos de controle é necessário obter a força externa tridimensional no espaço dos pontos de controle, designada como $F_{\text {ext }}^{p c}$, esta força decorre da transformação da força $F_{\text {ext }} 3 D$ para este espaço.

Considerando novamente a ilustração na Figura 5, onde um ponto $E$ da snake-3D é projetado nos pontos $E_{1}$ e $E_{2}$ sobre o par atual de mapas vetoriais, nota-se que esses pontos sofrem a ação das respectivas forças de imagem $F_{\text {ext } 2 D}\left(E_{1}\right)$ e $F_{e x t} 2 D\left(E_{2}\right)$, produzindo os pontos $F_{1}$ e $F_{2}$. Utilizando as matrizes de projeção, esses pontos são retroprojetados (Ayache [23]) de volta ao espaço 3D, gerando o ponto $F$. O vetor $\overline{E F}=F-E$ corresponde à força $F_{\text {ext }-3 D}$ procurada. 


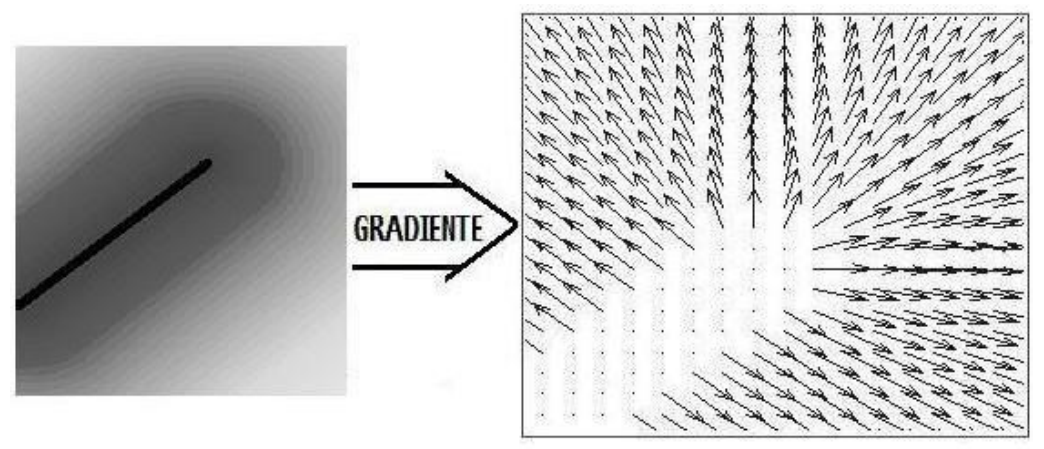

A

B

Figura 4. A) Imagem de um mapa de distâncias, onde a distância do pixel até o eixo-médio é traduzida em um nível de cinza. B) O campo de forças obtido a partir da aplicação do operador gradiente.

Segundo Cañero [20], a partir do vetor $F_{\text {ext } 3 D}$ aplicado na Equação 9 (onde $\mathbf{N}^{t}$ é a matriz de funções de base da $B$-spline $Q$ ), determina-se a direção $g(Q)$ do respectivo vetor $F_{\text {ext } 3 D}^{p c}$ de força externa no espaço dos pontos de controle, este, age sobre o ponto de controle $V$, a ele associado, modificando localmente a configuração da snake-3D. Esse processo se repete para os demais pontos de controle determinando o deslocamento do contorno ativo.

$$
\begin{gathered}
g(Q) \approx \mathbf{N}^{T} F_{\text {ext }-3 D}(Q) \\
F_{\text {ext } 3 D}^{c p}=\overline{\gamma V g(Q)}=g(Q)-V
\end{gathered}
$$

Os pontos da snake-3D representada pela $B$-spline são resultantes da combinação baricêntrica de pontos de controle. No caso da $B$-spline de terceira ordem utilizada nessa proposta, para cada intervalo no espaço paramétrico (span ou vão) os pontos do contorno ativo resultam da influência de três pontos de controle consecutivos e respectivas funções de base associadas, na Figura 6-A tais funções correspondem a $N_{i}(s)$. No entanto, essa influência é variável e sempre há um ponto de controle mais predominante na determinação de um ponto da curva, dentro do vão considerado. Por exemplo, considerando a Figura 6-A, nota-se que o ponto de controle que mais influência na criação do ponto $Q\left(s_{j}\right)$ é $V_{3}$, pois a função de base $N_{3}\left(s_{j}\right)$ possui valor mais alto do que as demais funções não nulas influentes no vão em questão, quais sejam $N_{2}\left(s_{j}\right)$ e $N_{4}\left(s_{j}\right)$. A Figura 6-B exibe uma curva gerada através dos pontos de controle de uma $B$-spline.

Utiliza-se a Equação 11 (Cañero [20]) para obter o conjunto de pontos do contorno ativo Pts associados aos respectivos pontos de controle, onde $\mathbf{N}$ corresponde à matriz de 

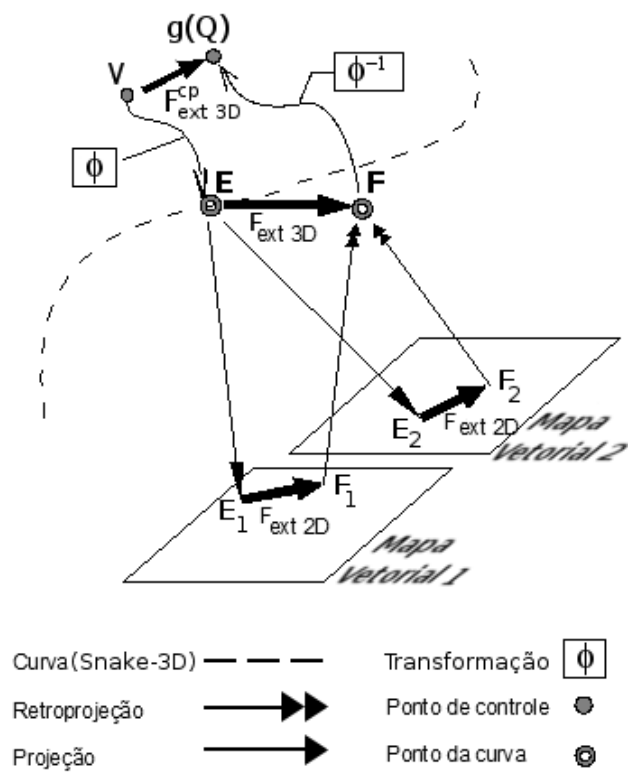

Figura 5. Composição da força externa $3 D$ a partir de dois mapas vetoriais e conversão desta para espaço de pontos de controle na forma de $g(Q)$. A transformação $\Phi^{-1}$ equivale à Equação 9 de conversão ao espaço dos pontos de controle, $\Phi$ equivale à Equação 11 a qual determina os pontos do contorno ativo.

funções de base e $\mathbf{V}$ ao conjunto de todos os pontos de controle.

$$
\text { Pts } \approx \mathbf{N} \text { V }
$$

Ainda sobre a Figura 5, note que os pontos $E_{1}$ e $E_{2}$ são correspondentes pois decorrem da projeção de um único ponto tridimensional (ponto $E$ da snake-3D), além disso, o modelo proposto também considera correspondentes os pontos $F_{1}$ e $F_{2}$ pois são deslocamentos dos respectivos $E_{1}$ e $E_{2}$ sob a ação de forças de imagens.

Observe que as coordenadas dos pares de pontos correspondentes $\left(E_{1}, E_{2}\right)$ e $\left(F_{1} e F_{2}\right)$ são determinadas sem a necessidade de qualquer procedimento adicional específico para detectá-los, por conta disso, se afirma que o modelo snake-3D não é afetado pelo problema da detecção de pontos homólogos/correspondentes.

Em conformidade com o conjunto de forças envolvidas, o processo de minimização conduz a snake- $3 D$ a uma estabilidade de energia, o que equivale a uma situação espacial 

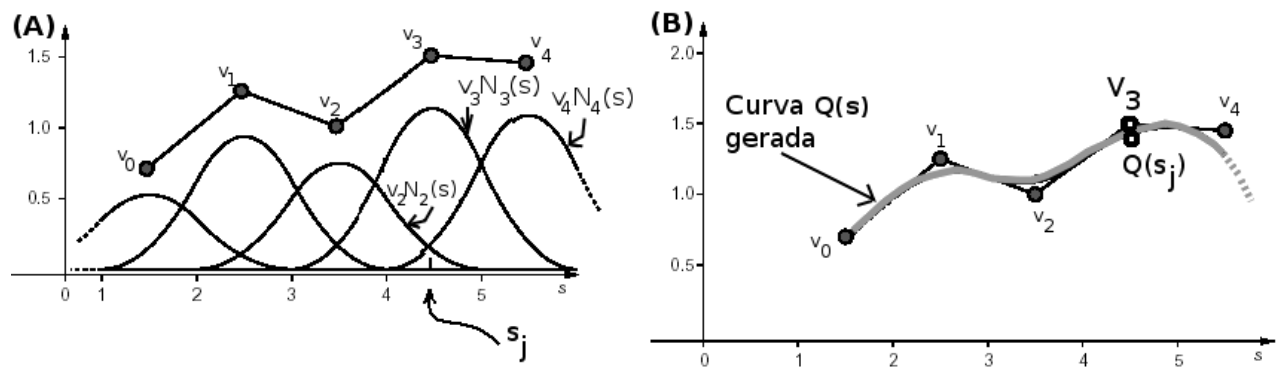

Figura 6. Considere $V_{i}$ como um de controle da $B$-spline $Q(s)$ de ordem três, com funções de base $N_{i}(s)$. Para cada vão (span), os pontos da curva $Q(s)$ (B) resultam da combinação dos três pontos de controle dominantes e funções de base associadas. Porém, a influência de cada ponto de controle varia e um deles pode predominar sobre os demais, é o caso do ponto $Q\left(s_{j}\right)$. Nota-se na parte superior (A) da figura que o ponto $Q\left(s_{j}\right)$ é mais influenciado pelo ponto de controle $V_{3}$, pois a função de base $N_{3}(s)$ é preponderante em relação às outras duas funções $\left(N_{2}(s)\right.$ e $N_{4}(s)$ ) desse vão. Adaptado de Blake e Isard [19].

ajustada ao eixo-médio do arco no espaço capturado no par de imagens correntes.

\subsection{Procedimento de Relaxação do Modelo}

A Figura 5 descreve o processo de ajuste de um ponto de controle, para o ajuste global da snake-3D é necessário considerar o conjunto $\mathbf{V}$ de todos os pontos de controle no procedimento de relaxação. Para realizar essa minimização utiliza-se o método numérico direto descrito na Equação 12, na qual o vetor $\mathbf{V}_{t}$ corresponde ao vetor de pontos de controle da $B$-spline que representa a snake-3D no instante $t$, o qual é determinado a partir do vetor $\mathbf{V}_{t-1}$ sob a ação do vetor de forças determinado pela Equação 13, na qual, $\mathbf{g}\left(\mathbf{Q}_{t-1}\right)$ corresponde a todas as direções das forças externas em $\mathbf{F}_{e x t}^{p c} 3 D^{2}$.

Ainda na Equação 12, a matriz $\mathbf{H}$ representa a rigidez do modelo, incorporando os parâmetros $(\alpha, \beta)$ os quais ponderam as forças internas controladoras da capacidade do contorno ativo se dobrar, se alongar ou se contrair. Por sua vez, a matriz I corresponde à matriz identidade, enquanto que o parâmetro $\gamma$ representa o passo temporal no processo de diferenciação implementado (Cañero [20]). Na Equação 12, o valor de $\mathbf{F}_{e x t}^{p c} 3 D$ é determinado através da Equação 13.

$$
\begin{gathered}
\mathbf{V}_{t}=\mathcal{F}\left(\mathbf{F}_{\text {int }}, \mathbf{F}_{\text {ext }}^{p c}, \mathbf{V}_{t-1}\right)=(\mathbf{H}+\gamma \mathbf{I})^{-1} \mathbf{F}_{\text {ext } 3 D}^{p c} \\
\mathbf{F}_{\text {ext } 3 D}^{p c}=\gamma \mathbf{V}_{t-1}-\mathbf{g}\left(\mathbf{Q}_{t-1}\right)
\end{gathered}
$$




\section{Inicialização da snake-3D}

A inicialização do modelo consiste em determinar a primeira $B$-spline a partir de um conjunto de pontos tridimensionais recuperados do primeiro par de imagens, para esse fim, tira-se proveito da geometria inicial do arco. Neste trabalho os arcos são gerados através da aplicação de tensão elétrica nos extremos de um fio fusível instalado ao longo de uma estrutura retilínea chamada cadeia de isoladores, por conta disso, nos seus primeiros instantes o arco é um objeto brilhante retilíneo cujas projeções também são retilíneas (conforme exibido na Figura 2-A). Tal aspecto facilita a determinação de homólogos/correspondentes. Aplicando a restrição imposta pelas retas epipolares, tal como descrito em Santos et al. [15], um ponto de um dos eixos retilíneos possui seu homólogo no cruzamento da respectiva reta epipolar com o outro eixo retilíneo. A partir do conjunto de pares de pontos correpondentes basta retroprojetá-los [23] para obtenção do conjunto inicial de pontos $3 D$ sobre os quais deve ser aplicada uma $B$-spline aproximadora, determinando a inicialização da snake-3D. Note que a determinação de homólogos é facilitada e necessária apenas neste momento de inicialização.

\section{Ajuste e Medição da Snake-3D}

Inicializado o modelo, o funcional de energia tem que ser otimizado sob a regulação das forças externas e internas ao contorno ativo de maneira que, para cada $i$-ésimo par de imagens, ocorra um estágio de ajuste $e_{i}$ no qual a snake-3D evolui de uma configuração inicial $Q_{i}$ para uma configuração final $Q_{i+1}$. Essa configuração final do modelo deve apresentar uma energia total mínima quando as projeções do contorno ativo se ajustarem em relação ao respectivo par de eixos-médios obtidos na fase de processamento de imagens.

Considerando a inicialização do modelo para o primeiro par e a disponibilidade da sequiência de pares de imagens, é possível utilizar a saída de um estágio como entrada do seguinte e, desta forma, capturar a evolução do arco no decorrer do tempo, bem como as medições ao final de cada estágio de evolução.

Uma vez obtida a configuração final $Q_{i+1}$ na saída do estágio $e_{i}$, basta executar uma medição da snake- $3 D$, para tanto, uma estratégia simples consiste em gerar a curva tridimensional equivalente à snake-3D (Algoritmo de D'Boor) e, em seguida, realizar o somatório das distâncias euclidianas entre pontos consecutivos dessa curva. O processo de rastreio e medição é sintetizado no fluxograma exibido na Figura 7. 


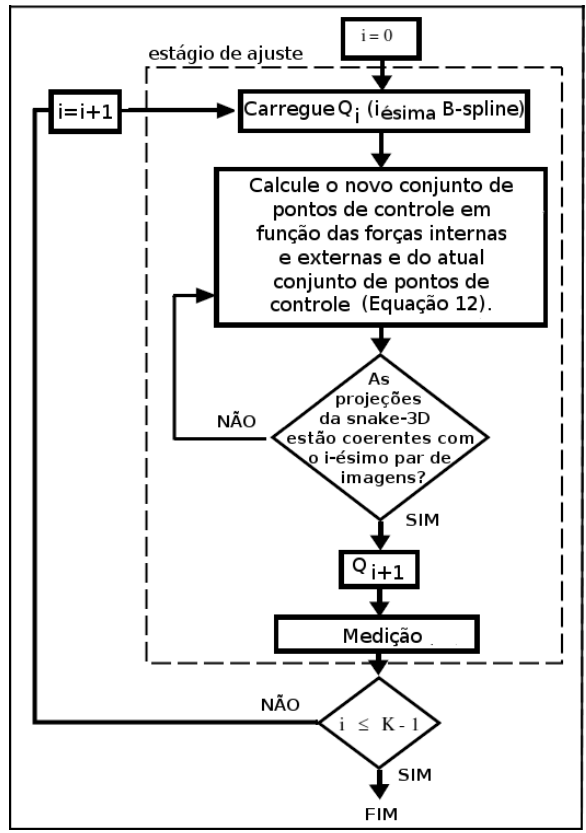

Figura 7. Fluxograma do processo de rastreio e medição para K pares de imagens.

\section{Calibração das Câmeras}

Conforme já foi evidenciado, a aplicação da proposta necessita do conhecimento das matrizes de projeção das câmeras, para tanto é preciso executar uma operação de calibração para cada câmera do sistema. Considerando a torre de energia (Figura 8) localizam-se marcos cujas coordenadas 3D foram pré-determinadas, então, captura-se uma imagem destes marcos utilizando a câmera a ser calibrada, tal imagem deve apresentar no mínimo sete pontos visíveis correspondentes às projeções dos marcos. Deve-se, então, determinar as coordenadas de imagem desses pontos, o que é feito manualmente visto que a complexidade das estruturas das torres dificulta a aplicação de uma técnica de identificação automática. Uma vez obtidas tais coordenadas, a correspondência de cada ponto 3D com sua projeção fornece o sistema de equações cuja solução consiste na matriz de projeção da câmera $\left(M_{\text {proj }}\right)$, procurada. O algoritmo "computação de parâmetros da câmera" utilizado para determinar a matriz de projeção está descrito em Trucco e Verri [25] e é aplicado para a calibração desejada.

Sob pena de comprometer a calibração e conseqüentemente as medições de compri- 




Figura 8. Marcos de coordenadas 3D conhecidas utilizados para calibração.

mento realizadas, as posições de calibração e ajustes de parâmetros internos determinadas para as câmeras (distância focal, por exemplo) devem ser mantidas constantes no transcorrer da captura das imagens de ensaios. Além disso, considerando o fato dos ensaios ocorrerem em ambiente não confinado, o tempo de duração do arco elétrico deve ser curto, com isso deseja-se manter os fatores ambientais (luminosidade e temperatura, por exemplo) relativamente constantes durante a ocorrência do ensaio do arco. As restrições eletrotécnicas, tais como a sustentação da alta corrente necessária, já impõe um tempo de duração de no máximo 1 segundo.

\section{Experimentos com Curvas Analíticas de Comprimento Crescente}

Inicialmente foram realizados experimentos de validação utilizando curvas analíticas. Apesar de não esgotar todas as possíveis geometrias dos arcos elétricos produzidos nos ensaios no laboratório CEPEL, estas curvas apresentam importantes características observadas nos mesmos, quais sejam, a apresentação de uma configuração inicialmente retilínea e um crescente aumento do comprimento e da sinuosidade.

\subsection{Experimento com Hélices}

Uma das famílias de curvas testadas consiste em seqüência de hélices descrita na Equação 14, alguns membros desta família são exibidos na Figura 10.

$$
C(t)=\left\{\begin{array}{l}
x(t)=r(t) \operatorname{sen}\left(A * w_{1}(t)\right) ; \\
y(t)=v * A ; \\
z(t)=r(t) \cos \left(A * w_{2}(t)\right) ;
\end{array}\right.
$$


Onde:

1. $A=(0, \ldots, \theta) \mathrm{rad}$

2. $t_{j}=t_{j-1}+\varepsilon$, onde $\varepsilon$ é um valor pequeno, para garantir a evolução suave desejada;

3. $r(t)=t / 5 ; t \in t_{1}, \ldots, t_{\text {max }}$;

4. $w_{1}(t)$ e $w_{2}(t)$ fazem com que cada membro da família de hélices apresente uma maior ondulação em relação ao plano $Y Z$ do que em relação ao plano $X Z$;

5. O valor do raio $r(t)$ é crescente, portanto, cada membro da família de hélices apresenta um comprimento maior do que o anterior;

6. $y(t)$ é constante de maneira que a primeira curva-membro $C\left(t_{1}\right)$ seja o mais retilínea possível. Na proporção que $t$ cresce $x(t)$ e $z(t)$ tornam-se gradualmente mais expressivos, como resultado, cada novo membro da família de hélices é levemente mais ondulado e alongado que o anterior.

Um sistema de aquisição foi simulado e as imagens foram geradas a partir de cada curva tridimensional da família de curvas, os parâmetros da matriz de projeção do sistema foram estipulados. Uma seqüência de pares de imagens é construída através da projeção de uma curva $C(t)$ gerada através da Equação 14. Portanto, cada par de imagens $\left(I_{1}^{t}, I_{2}^{t}\right)$ captura um membro da família de hélices gerado a cada valor de $t$ (Figura 9). Uma vez produzidas as imagens das curvas, estas são processadas tal como descrito na Seção 3 e em seguida aplicado o modelo aqui proposto.

A primeira curva membro é retilínea e gradualmente se alonga e se torna helicoidal, conforme exibido na Figura 10. É importante observar que a família de curvas é constituída por curvas-membros de comprimento crescente. Para cada $t$, o valor verdadeiro do comprimento da cada curva $C(t)$ é calculado e armazenado no vetor $\mathbf{L}$ de medidas verdadeiras $\mathbf{L}=\left\{L_{1}, \ldots L_{\max }\right\}$. Por sua vez, para cada par de imagens $\left(I_{1}^{t}, I_{2}^{t}\right)$ executa-se a respectiva medição via snake-3D constituíndo o vetor $\mathbf{L}_{\text {snake }}=\left\{L_{1}^{\text {snk }}, \ldots, L_{\text {max }}^{\text {snk }}\right\}$. Através da Equação 15 é então calculado o percentual do desvio $\left(d_{t}^{\text {perc }}\right)$ entre o valor medido e o respectivo valor verdadeiro do comprimento da curva para cada $t$. A média de todos os desvios $d_{t}^{\text {perc }}$ fornece um dado acerca da acurácia das medições realizadas através do método proposto, no caso em questão este valor foi de $3 \%$.

$$
d_{t}^{\text {perc }}=\frac{\left|L_{t}-L_{t}^{\text {snk }}\right|}{L_{t}} * 100
$$



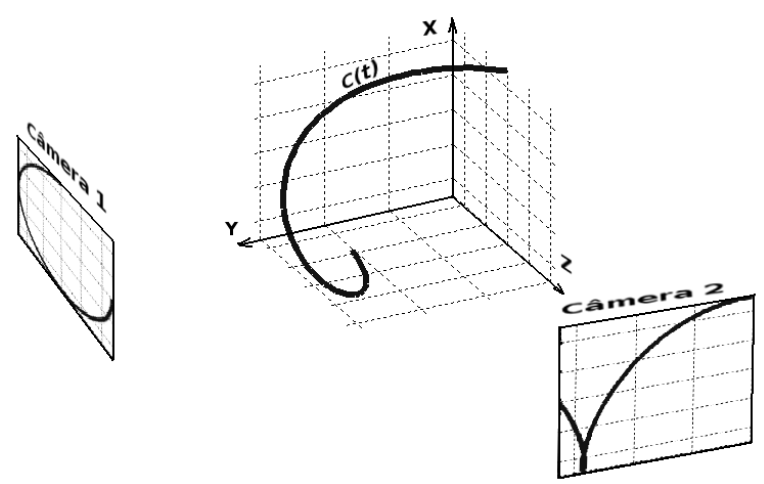

Figura 9. Um membro da família de hélices é capturado (projetado) nas imagens geradas pelo par de câmeras.

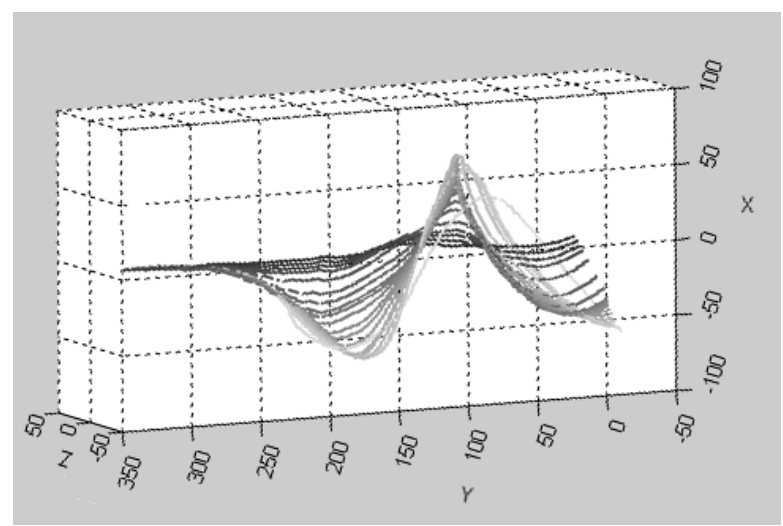

Figura 10. Exemplo de uma família de hélices. Cada curva membro se torna mais sinuosa a partir de uma configuração inicial retilínea (linha mais escura). Cada curva é gerada para um valor de $t$ na Equação 14.

\subsection{Experimento com Laços}

Não é incomum o surgimento de laços ao longo do arco elétrico, neste experimento é realizada uma simulação desse tipo de evento. Similarmente ao caso anterior, constrói-se uma família de curvas de maneira que cada membro desta família é projetado sobre um par de imagens. Novamente, a primeira curva apresenta-se retilínea e gradualmente as demais 

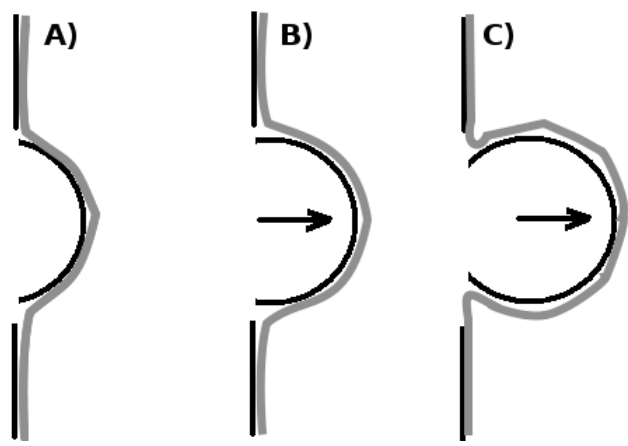

Figura 11. Formação de um laço: os pontos referentes a dois trechos de reta intercalados por um setor de circunferência são aproximados por uma $B$-spline formando a linha cinza que representa o laço crescente.

curvas formam o laço desejado.

Os pontos referentes a dois trechos de reta intercalados por um setor de circunferência são aproximados por uma $B$-spline que descreve a curva $C\left(\theta_{j}\right)$ resultante. Este processo é repetido com o setor de circunferência crescendo, vide a Figura 11. Os pontos do setor circular são determinados através da Equação 16, onde $\theta_{m}$ in $\leq \theta \leq \theta_{\max }$.

$$
\left(x_{j}, y_{j}, z_{j}\right)^{T}=\text { raio } *\left(\cos \left(\theta_{j}\right), \sin \left(\theta_{j}\right), 0\right)
$$

A Figura 12 exibe a família de curvas que formam o laço. Os comprimentos verdadeiros e medidos são tomados e é aplicada a Equação 15 para cada curva $C(\theta)$. Neste caso a média de todos os desvios $d_{t}^{\text {perc }}$ foi igual a $1,5 \%$.

\subsection{Análise de Resultados}

Há diversos fatores ambientais, climáticos e intrínsecos ao próprio arco os quais praticamente inviabilizam a reprodução, em curvas analíticas, de todas as formações possíveis de ocorrer em um arco elétrico em evolução em espaço aberto. No entanto, tais curvas propiciam a reprodução de algumas características importantes, as quais foram realizadas nos experimentos acima relatados. Ademais, não há perda expressiva de generalidade ao utilizar um sistema simulado de aquisição de imagens, visto que os fundamentos gerais da geometria projetiva foram preservados.

Para os experimentos com a família de hélices o valor do desvio percentual médio foi $d_{t}^{\text {perc }}=3,0 \%$ enquanto o caso da formação do laço apresentou $d_{t}^{\text {perc }}=1,5 \%$. O desvio mais alto 


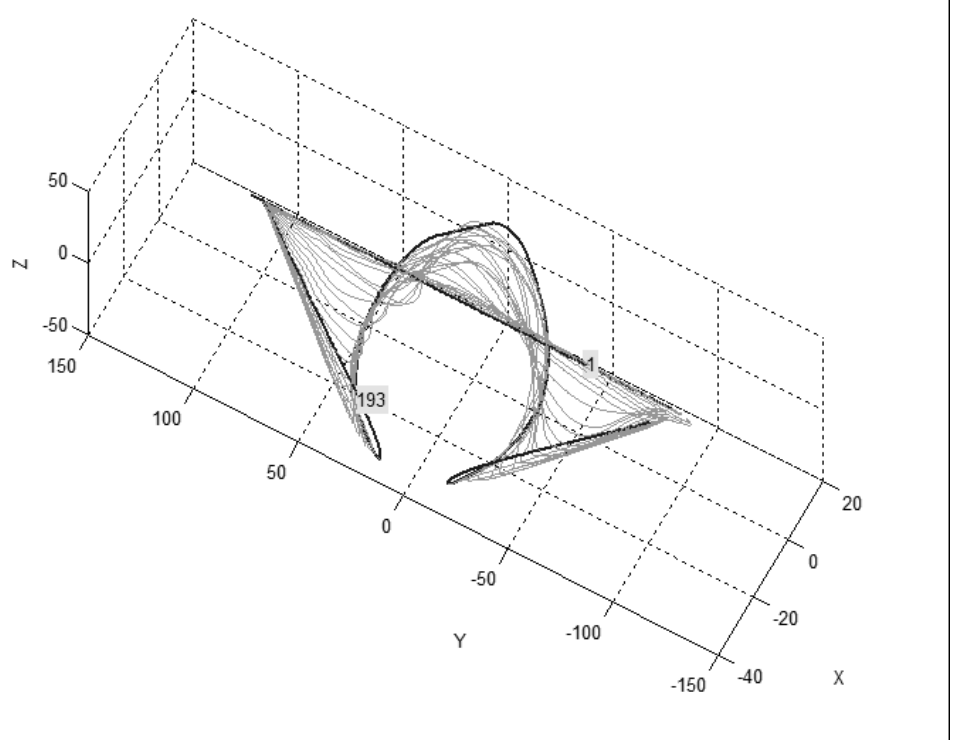

Figura 12. Família de curvas determinado um laço crescente.

obtido no experimento com hélices se deve ao forte momento torçor que atua ao longo da formação da hélice. O modelo snake-3D ora aplicado trata basicamente do momento fletor.

Não é usual a introdução de um componente de força interna de torção no modelo snake-3D pois levaria a um aumento indesejável no tempo de execução dos cálculos de minimização e exigiria recursos computacionais não disponíveis. Outro aspecto a analisar é que o arco elétrico não apresenta uma torção tão expressiva ao longo do seu eixo-médio, portanto, a família de hélices estressou o modelo e este respondeu com um desvio de 3\% o que não é numericamente alto e é aceitável para a aplicação de medição desejada.

Para o caso da família de curvas que formam o laço o valor da média de todos os desvios $d_{t}^{\text {perc }}$ foi igual a $1,5 \%$, este valor é numericamente baixo, comprovando a capacidade da abordagem proposta em atender à aplicação desejada.

Nenhum modelo matemático é ideal havendo sempre uma tentativa de aliar eficiência e um nível aceitável de acurácia. Considerando tal aspecto e o fato dos resultados decorrerem de comparação com os valores reais dos comprimentos das curvas, para o estágio atual de trabalhos os pesquisadores avaliaram os resultados como aceitáveis. 




Figura 13. Duas seqüências de imagens (A e B) de dois vídeos sincronizados com base no primeiro par de quadros (tempo $t_{i}$ ) quando o arco aparece.

\section{Experimentos com Arcos Elétricos Reais}

Aqui são apresentados dois experimentos com arcos elétricos gerados em torres de $500 \mathrm{kV}$. As capturas de imagens foram realizadas por um par de câmeras "domésticas" do tipo handycam, modelo Sony ${ }^{T . M . ~ H D R S R 10, ~ c o m ~ v e l o c i d a d e ~ d e ~} 30$ quadros por segundo, provendo imagens com resolução final de $720 \times 570$ pixels. Estas câmeras não possuem dispositivo eletrônico para o acionamento sincronizado da aquisição de imagens, desta forma, o par de seqüencias de quadros foi sincronizado a partir da inspeção visual do primeiro quadro que exibe o arco (Figura 13). Esta estratégia certamente não propicia um sincronismo real, porém mitiga este problema permitindo a realização do experimento utilizando os recursos disponíveis. Foram determinadas as matrizes de câmeras (Seção 6) através da aplicação de método de calibração. A variação do comprimento do arco ao longo de sua evolução é calculado via snake-3D e apresentado em gráfico comparativo.

Não existe uma maneira direta para a medição do comprimento do arco elétrico, bem como não há disponibilidade do valor verdadeiro dos seu comprimento. Porém, dispõe-se de estratégia eletrotécnica baseada na análise de harmônicos (Camara [1, 2, 3]). Não sendo baseada na reconstrução 3D do arco, esta técnica não é capaz de estabelecer o valor métrico do comprimento (ou da variação do comprimento). Apesar disso, ela é capaz de oferecer um perfil da curva $l(t)$ de variação do comprimento e na falta do valores verdadeiros dos comprimentos este perfil pode ser utilizado em comparações com as medições via snake-3D.

É de conhecimento (Camara [1, 2, 3]) que há uma relação entre o valor eficaz da 




Figura 14. Ensaio $\mathrm{n}-$ 1, em um mesmo gráfico: medições elétricas em $\mathrm{kV}$ e medições via snake-3D em metros. A região em destaque na curva de tensão representa os instantes de perturbação nos dados elétricos relativos à medição de tensão.

primeira pseudo-harmônica ${ }^{5}$ dos sinais de tensão e corrente medidas nas extremidades do arco (Camara [1, 2, 3]) da tensão entre os terminais do arco, $V 1_{e f}$, e seu comprimento $L$, tal que $\frac{V 1_{e f}}{L}=$ constante. Desta forma, a menos de um fator de escala, a curva de medições dos comprimentos do arco via reconstrução tridimensional tem um perfil parecido com o perfil da curva de medições da primeira pseudo-harmônica da tensão elétrica.

Os resultados obtidos nos dois ensaios identificados como $\mathrm{n}-1$ e $\mathrm{n}-2$ estão respectivamente exibidos nos gráficos contidos na Figura 14 e na Figura 15.

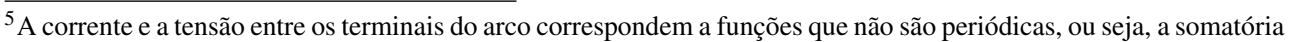
de senóides que as compõem têm amplitudes que aumentam ao longo do tempo. Portanto, não é possível obter o conteúdo harmônico estrito desses sinais. Por outro lado, a variação na amplitude é lenta o suficiente para ser desprezada dentro de um ciclo. Com esta consideração, o conteúdo harmônico de cada ciclo é calculado e um novo ciclo é analisado. Esta técnica de "janela deslizante" pode ser aplicada à tensão e à corrente, fornecendo as sequiências de conteúdos harmônicos para cada janela de tempo. Ao juntar estas seqüencias formam-se os conteúdos pseudo-harmônicos da tensão e da corrente do arco.
} 


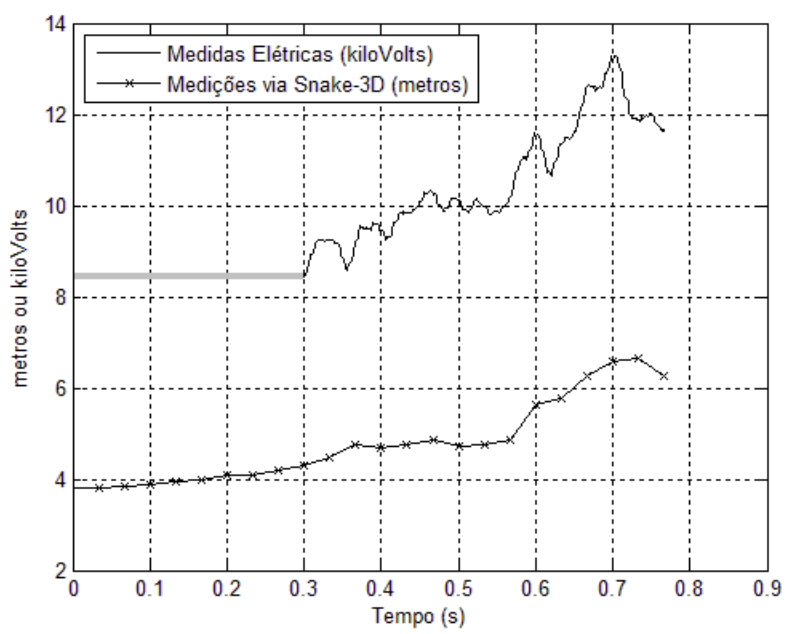

Figura 15. Ensaio $\mathrm{n}^{\circ} 2$, em um mesmo gráfico: medições elétricas em $\mathrm{kV}$ e medições via snake-3D em metros. A região em destaque na curva de tensão representa os instantes de perturbação nos dados elétricos relativos à medição de tensão.

\subsection{Análise de Resultados}

8.1.1 Ensaio no 1 Na Figura 14, em um mesmo gráfico sao exibidas as medições elétricas (medição do valor eficaz da primeira pseudo-harmônica da tensão) juntamente com as medições obtidas via snake-3D. Não há uma justaposição das duas curvas pois trata-se de duas ordens de grandeza diferentes (dezenas de milhares de volts versus poucas dezenas de metros). A medição de tensão ocorre a cada $40 \mu s$, portanto, em uma frequência bem maior (mil vezes) do que a taxa de amostragem das cameras de video, da ordem de $30 \mathrm{~ms}$ (a taxa de amostragem do modelo doméstico de câmera utilizado é de $30 \mathrm{fps}$, aproximadamente um quadro a cada $30 \mathrm{~ms}$ ). Esta diferença acaba afetando a acurácia da curva de variação do comprimento obtida através da snake- $3 D$ em comparação a curva obtida via medições elétricas. Observe que a cada $100 \mathrm{~ms}$ são realizadas 2.500 medições elétricas contra aproximadamente 4 capturas de pares de imagens. Este é um outro fator que influência a não ocorrência de um casamento perfeito entre as curvas obtidas via snake- $3 D$ em relação às curvas obtidas via medições elétricas.

Apesar das diferenças é possível notar, pela Figura 14, que os perfis das duas medições são semelhantes dentro do intervalo $[0,3-0,8]$ segundos. Neste intervalo as curvas são 
inicialmente quase horizontais apresentando pequenas oscilações, porém, sempre tendendo ao crescimento.

A curva de medição via snake- $3 D$ inicia em aproximadamente $4,0 \mathrm{~m}$, fato que é coerente com o comprimento da cadeia de isoladores que dá suporte ao fio fusível que faz a ignição do aro inicial.

A taxa de crescimento se acelera a partir de aproximadamente 0,7 segundos. Há uma tendência a um fator constante entre os valores correspondentes das duas curvas, ou seja, reproduz-se o fato da relação $V 1_{e f} / L$ ser aproximadamente constante.

Nos primeiros 0,3 segundos do ensaio o arco não se encontrava estabilizado e, até este instante, a primeira pseudo-harmônica da tensão não varia proporcionalmente ao comprimento do arco. Os valores associados ao intervalo $[0,0-0,3] \mathrm{s}$ foram descartados.

8.1.2 Ensaio no 2 São válidos os mesmos comentários feitos para o caso anterior. Os perfis das duas medições guardam semelhança, dentro do intervalo [0,3-0,75] segundos. Nos primeiros 0,3 segundos do ensaio o arco não se encontrava estabilizado e, ate este instante, a primeira pseudo-harmônica da tensão não varia proporcionalmente ao comprimento do arco. Os valores associados ao intervalo $[0,0-0,3] \mathrm{s}$ foram descartados.

\section{Conclusões}

No caso das curvas analíticas o método proposto apresentou medições com desvios considerados aceitáveis em relação aos valores verdadeiros dos comprimentos das curvas. No que se refere ao caso dos arcos reais, apesar do uso de câmeras domésticas de baixa velocidade e sem sincronismo automático (eletrônico), os resultados se mostraram consistentes com estudos encontrados na literatura (Camara $[1,2,3]$ ) os quais são baseados na análise de dados elétricos.

A abordagem descrita neste trabalho demonstra ser promissora e pode servir como ferramenta importante na medição de arcos para fins da modelagem matemática desejada. Ademais, considerando que a qualidade do sistema de aquisição de imagens tem influência significativa sobre os resultados da aplicação da abordagem proposta, por exemplo, no refinamento das forças externas que guiam a snake-3D, a utilização de um sistema que atenda aos requisitos necessários (sincronismo eletrônico, alta taxa de amostragem na aquisição de imagens, alta resolução espacial e fotométrica dos sensores) propiciará melhores resultados nas medições.

Uma outra contribuição do presente trabalho é demonstrar o potencial de aplicações de visão por computador no campo da eletrotécnica, uma área ainda pouco explorada por soluções baseadas em visão computacional e processamento de imagens. 


\section{Agradecimentos}

Os autores agradecem à Universidade do Estado de Santa Catarina (UDESC), à CAPES , financiadora do projeto 2007/05881-4, bem como ao CNPq e à FAPESP. Os resultados referentes aos ensaios de arco elétrico são resultados parciais do projeto P\&D ANEEL dos anos 2001/2002 financiado por FURNAS Centrais Elétricas S.A. . O projeto foi coordenado pela COPPE/UFRJ com a participação da UNICAMP. Os trabalhos de laboratório foram realizados pelo CEPEL na linha experimental construída nas suas dependências.

\section{Referências}

[1] A. Câmara, C. Portela, M. Tavares. Single-Phase Auto-Reclosure Studies: Some Basic Aspects on Main Elements Representation. International Conference on High Voltage Engineering and Application, pages 482-485, 2008.

[2] A. Câmara, C. Portela, M. C. Tavares. Single-Phase Auto-Reclosure Studies Considering a Robust and Reliable Secondary Arc Model Based on a Gray-Box Model. International Conference on High Voltage Engineering and Application, pages 486-489, 2008.

[3] A. Câmara, R. Gonçalves, C. Portela, M. Tavares. Arc Model for Single-Phase AutoReclosure Studies: Research in an Experimental 500 kV Line. International Conference on High Voltage Engineering and Application, pages 490-493, 2008.

[4] C. Portela. Study of Problems Related to Switching of Relatively Small Currents. In CIGRÉ Proceedings, pages 1-14, 1980.

[5] E. Anke et al. Practical Applications of Arc Physics in Circuit Breakers. Survey of Calculation Methods and Application Guide. In CIGRÉ - ELECTRA, volume 118, pages 63-79, num. 0, 1988.

[6] C. Portela, C. Dupont, M. Meirelles. Deterministic and Statistic Arc Modeling. In CIGRÉ Proceedings, pages 1-6, 1994.

[7] C. Portela, N. Santiago, J. Mourente. Modelling of Dynamic Arc Behaviour for Small Currents. In CIGRÉ Proceedings - Colloquium of CIGRÉ Study Committee 13, pages 1-28, 1989.

[8] C. Portela, N. Santiago, O. Oliveira, C. Dupont. Modelling of Arc Extinction in Air Insulation. In Third International Conference on Properties and Applications of Dielectric Materials - ICPADM, pages 381-384, 1991.

[9] M. Tavares, C. Portela. Transmission System Parameters Optimization - Sensitivity Analysis of Secondary Arc Current and Recovery Voltage. IEEE Transactions on Power Delivery, 19(3):1464-1471, 2004. 
[10] J. M. K. MacAlpine, D. H. Qiu, and Z. Y. Li. An analysis of spark paths in air using 3-dimensional image processing. IEEE Transactions on Dieletrics and Electrical Insulation, 6(3):331-336, 1999.

[11] D. H. Qiu and J. M. K. MacAlpine. An incremental analysis of spark paths in air using 3-dimensional image processing. IEEE Transactions on Dieletrics and Electrical Insulation, 7(6):758-763, 2000.

[12] D. H. Qiu, J. M. K. MacAlpine, and Z. Y. Li. An incremental 3-dimensional analysis of spark paths in air. In Conference on Electrical Insulation and dieletric Phenomena, volume 2, pages 646-649, 1999.

[13] D. Amarasinghe, U. Sonnadara, M. Berg, and V. Cooray. Correlation between brightness and channel currents of electrical discharges. IEEE Transactions on Dielectrics and Electrical Insulation, 14(5):1154-1160, 2007.

[14] M. Sellathurai. A binocular stereo technique for 3-d reconstruction of electrical discharges. In Proceedings of International Conference on Image Processing, volume 3, pages 284-287, 1997.

[15] G. B. Santos, S. P. Cunha, C. Tozzi. Rastreamento de Curvas 3D através de Biplane Snakes. Iberian Latin American Congress on Computational Methods in Engineering (XXIX CILAMCE 2008), Macéio, Brasil, Nov. 2008.

[16] G. Santos, S. Cunha, C. Tozzi. A New Application for 3D-Snakes - Modelling Electrical Discharges. 4th International Conference on Computer Vision Theory and Applications (VISAPP 2009), Lisboa, Portugal, pg. 546-553, Fev. 2009.

[17] G. Santos, C. Tozzi, M. Tavares. Visual Evaluation of the Length of Artificially Generated Electrical Discharges By 3D-snakes. IEEE Transactions on Dielectrics and Electrical Insulation, Vol. 18, Issue 1, pp. 200-210, February 2011.

[18] M. Kass, A. Witkin and D. Terzopoulos. Snakes: Active contours models. Second International Conference in Computer Vision, 1(4):321-331, 1988.

[19] A. Blake and M. Isard. Active Contours. Springer, 2nd edition, 2000. Também disponível em http://research.microsoft.com/en-us/um/people/ablake/contours/último acesso em $03 / 02 / 2011$.

[20] C. Canero. 3D Reconstruction of the Coronary Tree Using Biplane Snakes. PhD thesis, Universitat Autònoma de Barcelona, Bellaterra, 2002.

[21] R. Gonzalez and R. Woods. Processamento de Imagens Digitais., chapter Segmentação de Imagens., pages 295-341. Edgar Blücher, 2000. 
[22] P. Soille. Morphological Image Analysis - Principles and Application., chapter Hit-orMiss and Skeletons., pages 129-151. Springer, 1999.

[23] N. Ayache. Artificial Vision for Mobile Robots., chapter Calibration, pages 13-42. MIT Press, 1991.

[24] A. Blake and M. Isard. Active Contours., Appendix A, pages 281-296. Springer, 2nd edition, 2000. Também disponível em http://research.microsoft.com/enus/um/people/ablake/contours/ último acesso em 03/02/2011.

[25] E. Trucco and A. Verri. Introductory techniques for 3D Computer Vision., chapter Camera Calibration., pages 123-137. Prentice Hall, 1998. 